\title{
Research Article \\ Effect of Ga on the Inoxidizability and Wettability of Sn-0.5Ag-0.7Cu-0.05Pr Solder
}

\author{
Xu Jiachen, ${ }^{1}$ Xue Songbai, ${ }^{1}$ Luo Dongxue, ${ }^{1}$ Wang He, ${ }^{1}$ and Xue Peng ${ }^{2}$ \\ ${ }^{1}$ College of Materials Science and Technology, Nanjing University of Aeronautics and Astronautics, Nanjing 210016, China \\ ${ }^{2}$ School of Materials Science and Engineering, Nanjing University of Science and Technology, Nanjing 210016, China \\ Correspondence should be addressed to Xue Songbai; xuesb@nuaa.edu.cn
}

Received 27 May 2017; Accepted 21 June 2017; Published 10 September 2017

Academic Editor: Patrice Berthod

Copyright (c) $2017 \mathrm{Xu}$ Jiachen et al. This is an open access article distributed under the Creative Commons Attribution License, which permits unrestricted use, distribution, and reproduction in any medium, provided the original work is properly cited.

\begin{abstract}
The effect of trace amount of $\mathrm{Ga}$ on the inoxidizability and wettability of $\mathrm{Sn}-0.5 \mathrm{Ag}-0.7 \mathrm{Cu}-0.05 \mathrm{Pr}$ solders was investigated systematically by means of microstructure characterizations. The results indicate that the wettability and oxidation resistance properties are remarkably improved with addition of trace amount of Ga. Moreover, it is observed that the trace amount of Ga in $\mathrm{Sn}-0.5 \mathrm{Ag}-0.7 \mathrm{Cu}-0.05 \mathrm{Pr}$ solders refines the matrix microstructure. The relationship between wettability and oxidation resistance was put into deep study. And Ga was found to be enriched on the surface of the molten solder, which benefited the properties correspondingly. The results of this study can stimulate the use of low-silver $\mathrm{Sn}-\mathrm{Ag}-\mathrm{Cu}-\mathrm{Pr}$ solders for various applications.
\end{abstract}

\section{Introduction}

With the promulgation of the WEEE and RoHS directives, electronic manufacturing has shifted to green manufacturing system of "lead-free assembly" [1]. As the extensions of $\mathrm{Sn}-\mathrm{Ag} / \mathrm{Sn}-\mathrm{Cu}$-based solders, $\mathrm{Sn}-\mathrm{Ag}-\mathrm{Cu}$ solder system is considered as the best choice to replace the traditional $\mathrm{Sn}-\mathrm{Pb}$ solder. Compared with the other binary and ternary lead-free solders, $\mathrm{Sn}$-Ag-Cu solder has good wettability and reliability, and its tensile strength is 1.8 times better than the conventional solders as well. In addition, it has excellent thermal fatigue performance and fine pitch soldering of electronic components and the bridging phenomenon can hardly happen.

The existing $\mathrm{Sn}-3.8 \mathrm{Ag}-\mathrm{Cu}$ lead-free solder cannot meet the new trend in the field of electronic packing due to the severe interface reaction, the negative effects of the brittle compound in the solder joints, and the high cost of $\mathrm{Ag}$ as well. Therefore, it is necessary to explore the Sn-Ag-Cu leadfree solder with low-Ag content. However, with the reduction of $\mathrm{Ag}$ content, the properties such as wetting behavior and thermal fatigue performance become worse [2-5].

$\mathrm{Ga}$ can be easily enriched on the surface of the $\mathrm{Sn}-\mathrm{Pb}$ solder liquid surface and form a dense protective film to improve the oxidation resistance of the solder [6,7]. Similarly, in this paper, $\mathrm{Ga}$ is added into the solder to ameliorate such problems. This paper aims to study the new low-Ag lead-free solder with a good prospect. By adding proper amount of alloying element $\mathrm{Ga}$, the microstructure and properties of the solder are optimized, which can be close to those of the Sn$3.8 \mathrm{Ag}$ - $\mathrm{Cu}$ solder, thereby reducing the manufacturing costs. The results of this paper have important practical values for the development of the electronic industry.

\section{Experimental Procedure}

2.1. Alloy Design and Preparation. $\mathrm{Sn}, \mathrm{Ag}, \mathrm{Cu}$, and $\mathrm{Ga}$ of 99.9\% purity were used to prepare the solder alloys, and the compositions of element Ga were shown in Table 1. The melting process was conducted under Nitrogen atmosphere at $350^{\circ} \mathrm{C}$ for several minutes while mechanical stirring was carried out every minute. After melting, the solder was cooled to room temperature and cut into bars of $40 \mathrm{~mm}$ length. The compositions of the solders were investigated by inductively coupled plasma emission spectrometer (ICP-AES).

2.2. Solderability Test. The solderability test was carried out with an SAT-5100 Solder Checker. Using the wetting balance 


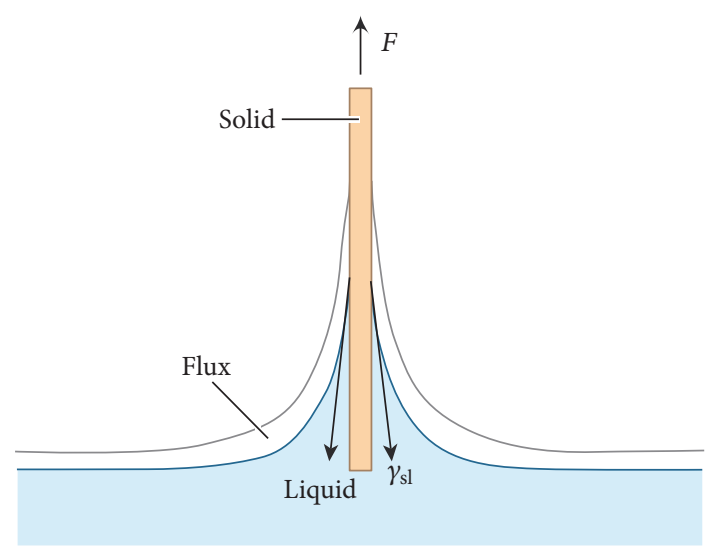

FIGURE 1: The stress situation of the $\mathrm{Cu}$ foil.

TABLE 1: Composition of solder alloy (wt.\%).

\begin{tabular}{lcccccr}
\hline & \multicolumn{6}{c}{ Solder alloys } \\
& 1 & 2 & 3 & 4 & 5 & 6 \\
\hline $\mathrm{Ga}$ (wt.\%) & 0 & 0.05 & 0.1 & 0.25 & 0.5 & 1 \\
Initial alloy & & $\mathrm{Sn}-0.5 \mathrm{Ag}-0.7 \mathrm{Cu}-0.05 \operatorname{Pr}(\mathrm{SACP} 0507)$ & \\
\hline
\end{tabular}

method, the wetting force and wetting time were recorded. For the advance preparation, the $\mathrm{Cu}$ foils $(30 \mathrm{~mm} \times 5 \mathrm{~mm} \times$ $0.3 \mathrm{~mm}$ ) were cleaned in ethanol and dried. The temperatures were set as $245,255,265$, and $275^{\circ} \mathrm{C}$ and water-soluble flux was chosen. The stress situation of the $\mathrm{Cu}$ foil in the wetting process was shown in Figure 1. Each test was conducted 5 times and the average result was obtained for analyzing.

2.3. Microstructure. The microstructure was examined by optical microscope and scanning electron microscope (SEM). A solution of $5 \% \mathrm{HNO}_{3}$ and $95 \%$ alcohol was configured to etch the samples. Energy Dispersive X-ray Spectrometry (EDS) was adopted to identify the phase structure of the solder.

2.4. Oxidation Resistance Test. The surface oxide film of the solder after a certain time of heat preservation can visually estimate the degree of the solder oxidation. Different components of the solders were melted on the solderability tester and kept at $245^{\circ} \mathrm{C}$ for $30 \mathrm{~min}$, recording its surface oxide film morphology.

To further investigate the oxidation resistance of the solder, high-temperature oxidation weight gain method was used. Solders of different components were rolled into solder sheets of about $0.15 \mathrm{~mm}$. After grinding and polishing to remove the oxide film, the solder was cleaned with ethanol and dried to produce small square pieces of $0.1 \mathrm{~mm} \times 10 \mathrm{~mm} \times$ $10 \mathrm{~mm}$. The samples were put into a furnace at $245 \pm 5^{\circ} \mathrm{C}$ and removed to cool every 10 hours till 60 hours. Analytical balance was used to weigh the total weight of the solder and the crucible. The resulting data was analyzed to show the growth of the sample mass.
TABLE 2: Melting point of the SACP-xGa $\left(\mathrm{T} /{ }^{\circ} \mathrm{C}\right)$.

\begin{tabular}{lcc}
\hline & SACP-0Ga & SACP-1Ga \\
\hline Solidus Temperature & $216.8^{\circ} \mathrm{C}$ & $211.3^{\circ} \mathrm{C}$ \\
Liquids Temperature & $227.2^{\circ} \mathrm{C}$ & $225.6^{\circ} \mathrm{C}$ \\
\hline
\end{tabular}

\section{Results and Discussion}

3.1. Microstructure. Figure 2 showed the microstructure of as-soldered SACP0507-xGa solders $(x=0,0.05,0.1,0.25,0.5$, $1)$. The microstructure of the SACP0507 solder was shown in Figure 2(a). $\beta$-Sn, eutectic region, and intermetallics were notified separately and it is easy to discover that $\beta$-Sn took the largest proportion and the IMC particles and eutectic were dispersed within Sn-rich matrix. According to the previous researches $[8,9]$, the IMC particles were mainly $\mathrm{Cu}_{6} \mathrm{Sn}_{5}$ and $\mathrm{Ag}_{3} \mathrm{Sn}$. Compared with the high-silver solder, the content of the brittle $\mathrm{Ag}_{3} \mathrm{Sn}$ in the matrix was reduced. These large particles and $\beta$-Sn grain always appeared in low-Ag SACP solder, which might be a threat to the premature failure [1013]. The melting point was shown in Table 2. The results indicated that the effect of Ga on the melting point of SACP lead-free solder is slight.

As the content of $\mathrm{Ga}$ element gradually increased, the matrix structure refined and the coarse IMC matrix particles became smaller and distributed more uniformly and appeared to be ellipsoidal-shaped instead of long-stripshaped. When the Ga content was $0.5 \mathrm{wt} . \%$, a uniform and fine microstructure is obtained and the grain reached the highest degree of refinement. The IMC particles in the SACP$0.5 \mathrm{Ga}$ solder were smaller, with respect to the SACP solder. On the basis of the strengthening principle, the mechanical properties of the solder could also be enhanced. However, as the amount of Ga increased to $1 \mathrm{wt} . \%$, some new phases with large size and nonuniform distribution could be found. The compositions were analyzed by EDX, the result was shown in Figure 3. It could be seen that the phase was mainly composed of $\mathrm{Sn}, \mathrm{Cu}$, and $\mathrm{Ga}$.

As shown in Figure 4 of the Ga-Sn binary phase diagram [14], as the content of Ga element is less than its maximum solubility in $\mathrm{Sn}$ which is $6.4 \%, \beta$-Sn mainly exists in the form of solid solution at room temperature.

3.2. Wettability. Ga element can decrease the surface tension of alloys [15]. In this paper, the improvement of the wetting ability due to $\mathrm{Ga}$ addition was reported. Wetting balance method was used and the wetting forces and wetting time of solders with different content of $\mathrm{Ga}$ at different temperatures were tested and the results were shown in Figure 5. At certain temperature, according to the test results, with the Ga content increasing, the wetting force significantly increased, while the wetting time showed a relatively large reducing tendency. And the best wettability was achieved when the content of Ga was $0.5 \%$, and then the wettability showed a slight downward trend when the addition kept increasing, suggesting that the proper addition of Ga was about 0.5 wt.\%.

The good wetting properties of the solder are a prerequisite for good solder joints, which is directly related to the 


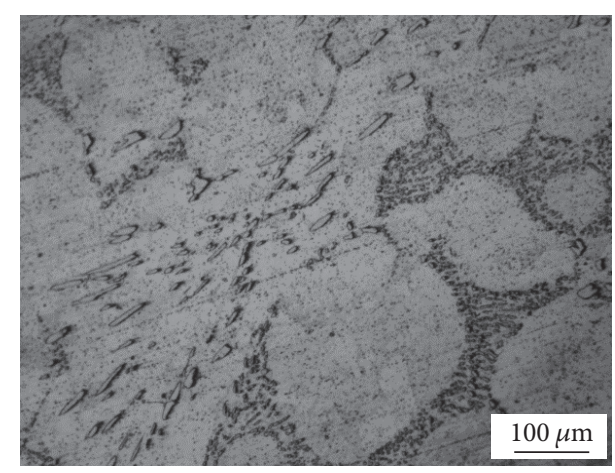

(a) SACP-0Ga

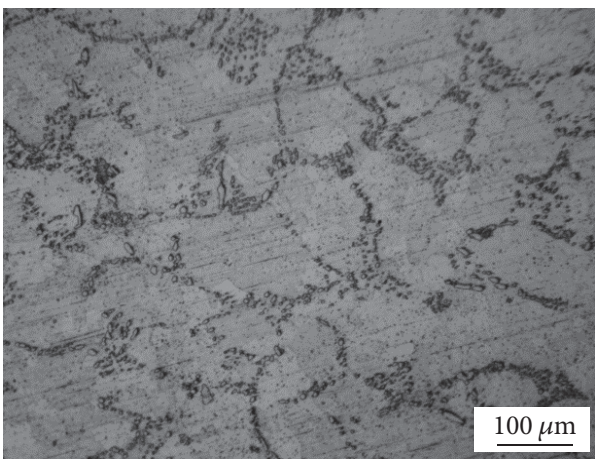

(c) SACP-0.1Ga

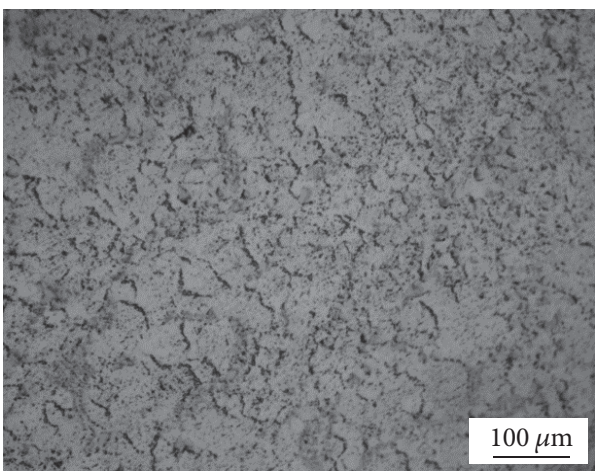

(e) SACP- $0.5 \mathrm{Ga}$

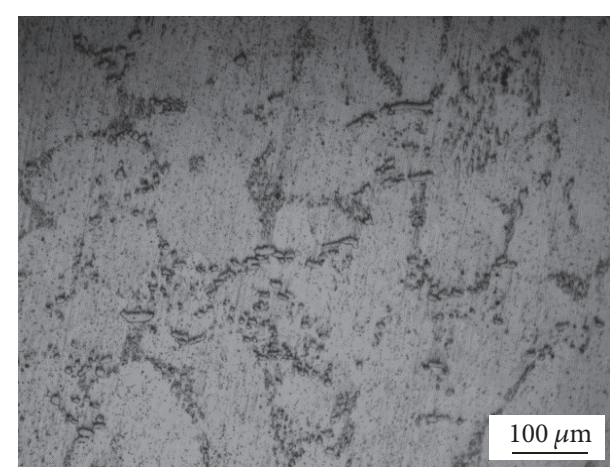

(b) SACP-0.05Ga

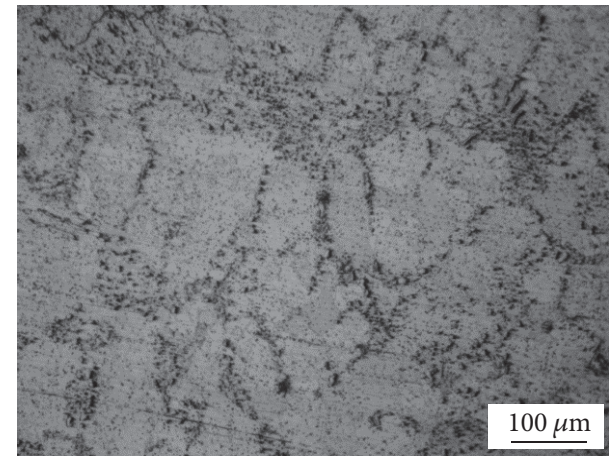

(d) SACP- $0.25 \mathrm{Ga}$

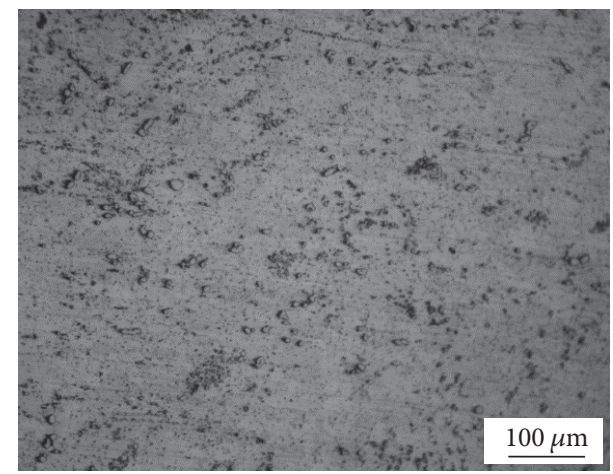

(f) SACP-1Ga

FIgURE 2: The microstructure of SACP0507- $x$ Ga solders.

reliability of the solder joints; therefore the wettability of the soldering process is listed as an important factor [15]. Figure 6 showed the wetting angles of the liquid solder at different wetting conditions. It can be seen directly that the smaller the value of $\theta$ is, the better wetting properties the solder has, as the Young-Dupre formula shows:

$$
\sigma_{\mathrm{gs}}=\sigma_{\mathrm{ls}}+\sigma_{\mathrm{gl}} \cos \theta
$$

where $\theta$ represents the wetting angle and $\sigma_{\mathrm{gs}}, \sigma_{\mathrm{gl}}$, and $\sigma_{\mathrm{ls}}$ represent the interfacial tensions between the solid-gas, liquid-gas, and liquid-solid interfaces separately.

Typically, the compositions and the surface tension of the solder and the base metal are two important factors that affect the solder's wettability. Under certain circumstances that the compositions are identified, the surface tension of the solder and base metal plays a key role in the wettability of the solder, and its influence factors are temperature, flux, metal surface oxides and surface active substances, and so forth. The copper used was deoxygenated, and the flux was the same, so it could be estimated that the surface active substances were added to improve the solder's wettability by way of reducing the surface tension.

Element $\mathrm{Ga}$ is a surface active element and strong positive adsorption can take place. As a result, the composition and structure of the liquid solder surface are changed, the interfacial tension is reduced, and the flowability is significantly improved, causing the refinement of wetting process of the liquid solder. In this paper, when the Ga content exceeded 


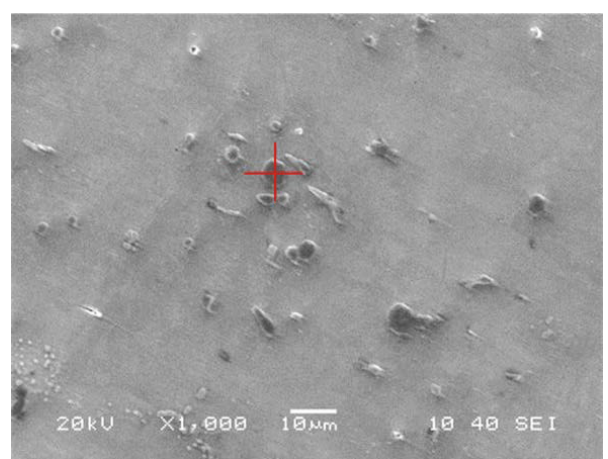

(a)

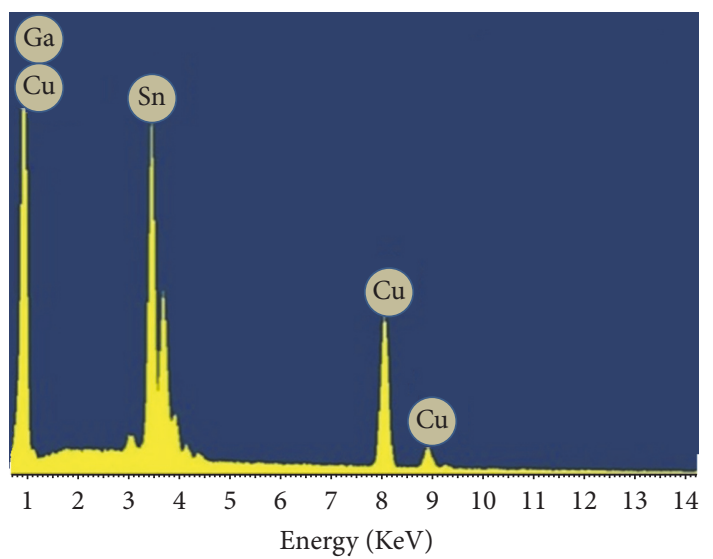

(b)

FIGURE 3: The EDX result of SACP0507-1Ga solder.

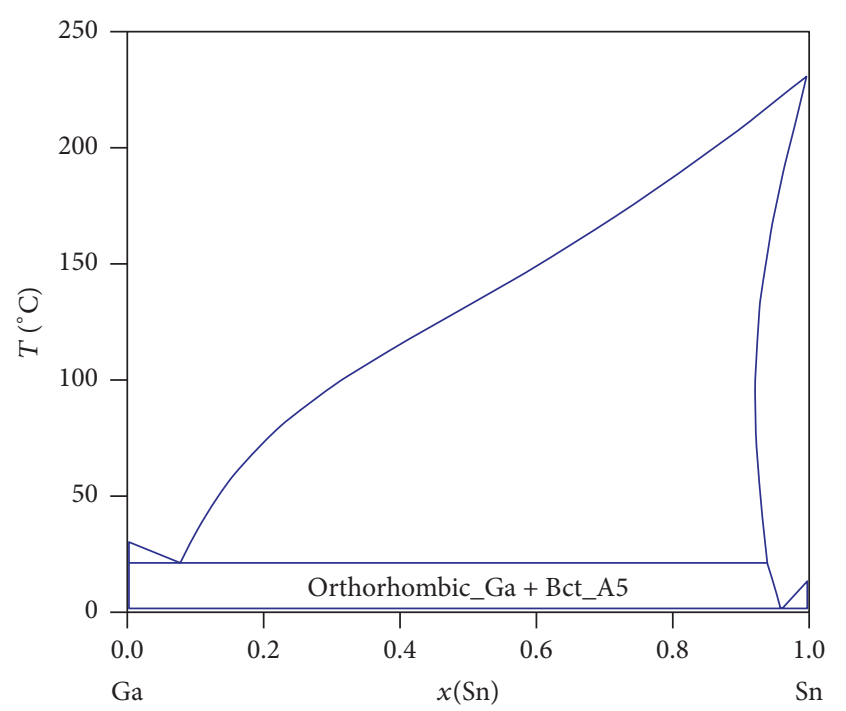

Figure 4: The Ga-Sn binary phase diagram.

$0.5 \%$, the surface enrichment got relatively stable, so the wettability no longer rose. However, the generation of the $\mathrm{Ga}$ rich phase limited the active effect of Ga element, decreasing its improvement of the solder's wetting properties.

3.3. Oxidation Resistance. The oxidation of solder in molten state is a problem to be faced; the generated oxides have adverse factors in the wettability and reliability of the solder joints in the service process. Under the protection of Nitrogen atmosphere, the oxidation resistance ability can increase, while, in actual industrial production, it requires optimization in the complexity and cost of the equipment and technology, which is not feasible [16]. Early studies show that $\mathrm{Ga}$ is highly enriched on the surface of $\mathrm{Sn}-\mathrm{Pb}$ solders and the degree of Ga enrichment can rapidly reach 34000-fold for a nominal additive amount [17]; the enriched Ga can form a protective film to prevent the oxidation of the solder alloy. In this chapter, the improvement in oxidation resistance at high temperature as a result of $\mathrm{Ga}$ addition has been studied.

\subsection{Surface Morphology after High-Temperature Oxidation.} To simulate the reflow process condition, the temperature was set at $245^{\circ} \mathrm{C}$. The solder was in molten state and the surface atoms were prone to react with oxygen in the air so that the quality of the solder changed. Therefore, difference in the change in the solder's quality could be a factor to characterize the difference between the oxidation resistance properties of the solders. Figure 7 showed the surface morphology photographs of several chosen SACP0507-xGa solders after incubation at $245^{\circ} \mathrm{C}$ for $30 \mathrm{~min}$. As can be seen, the SACP0507 solder surface was covered with a thick layer of gray dense oxide slag. As the content of Ga exceeded, the thickness of the surface oxide film was thinner, and in SACP-0.5\%Ga solder the surface was brighter and basically could not see the oxide layer, which showed excellent oxidation resistance. However, when the content of Ga reached 1\%, colored oil slick existed on the surface of the solder.

3.5. Weight Gain after High-Temperature Oxidation. Hightemperature oxidation weight gain method is used to quantitatively analyze the oxidation resistance of the solders. The mass ratio of the oxidized solder weight gain was calculated to study the impact of law of element $\mathrm{Ga}$; the results were shown in Figure 8. From the comparison, for solders of certain $\mathrm{Ga}$ content, the oxidation degree of the solder increased in the oxidation process, but when the incubation time exceeded 30 hours, the trend of increase declined. In the same oxidation period, the weight gain of solder excluding $\mathrm{Ga}$ was the highest, indicating the oxidation resistance was the worst and an appropriate amount of Ga could improve the oxidation resistance of the solder. The oxidation rate of the solder decreased with increasing time and this was because a protective oxide was formed on the solder surface, inhibiting the further oxidation of the metal matrix efficiently. With the increase of $\mathrm{Ga}$ element in the solder, the oxidation resistance 


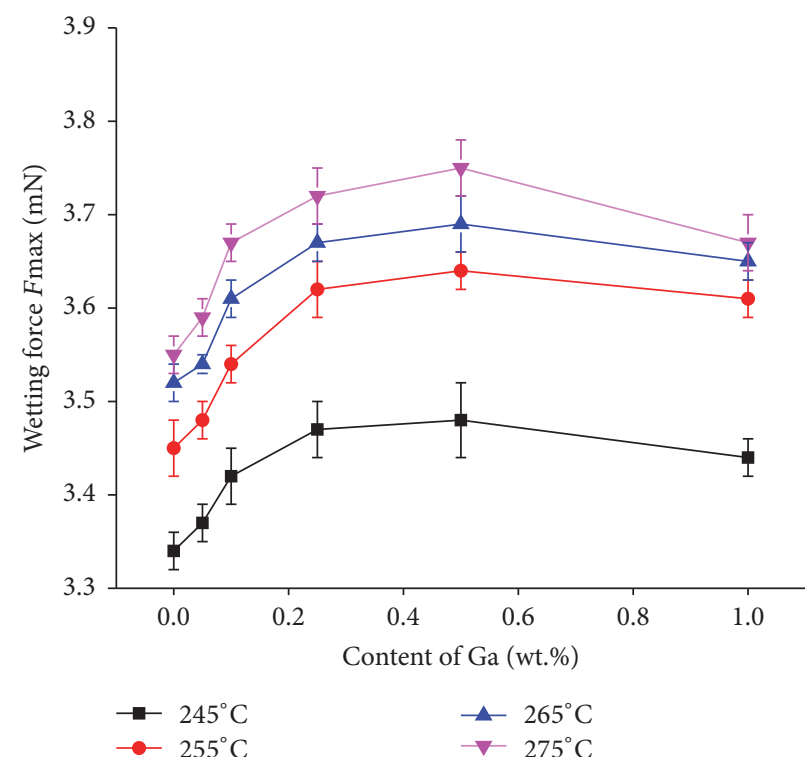

(a)

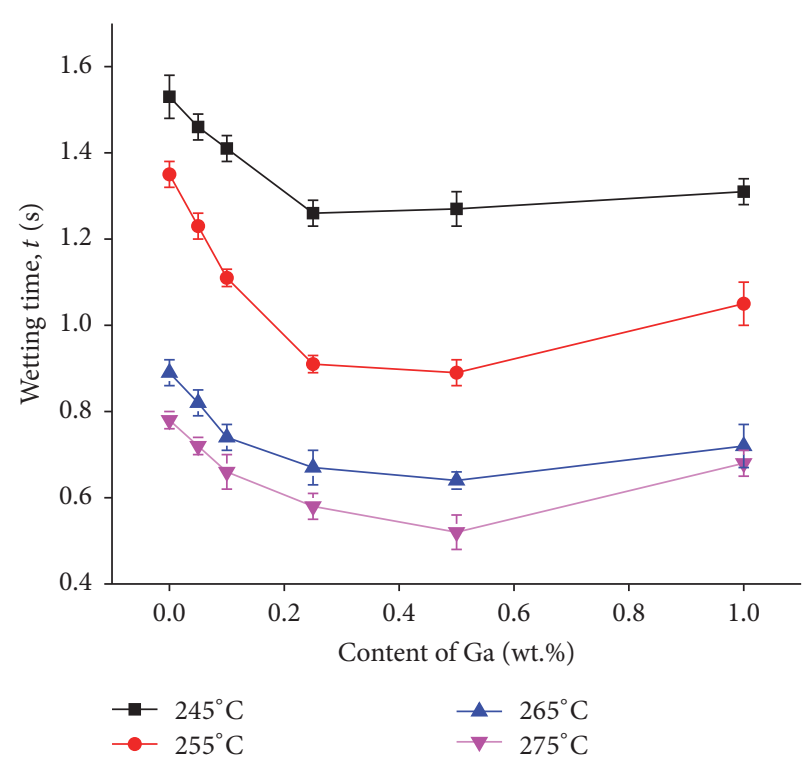

(b)

FIgURE 5: The wetting ability of the solders.

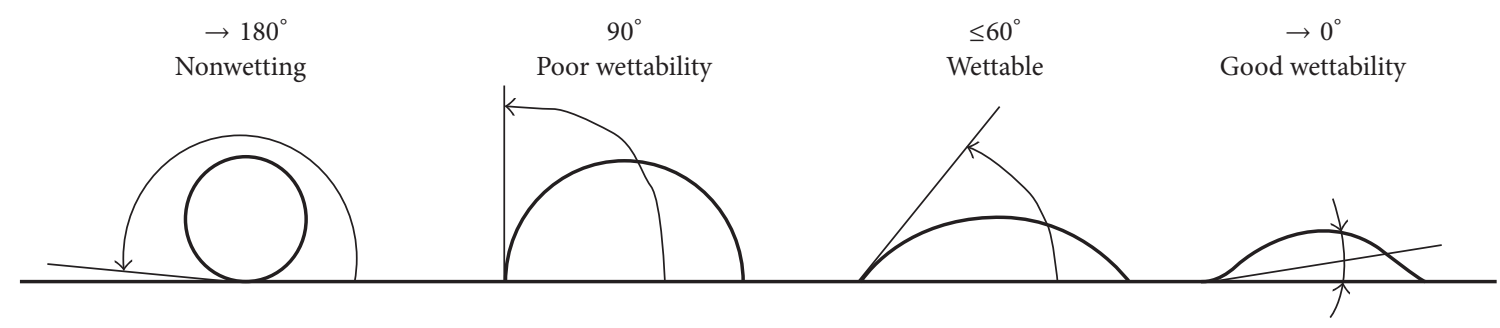

FIGURE 6: The wetting angles at different wetting conditions.

was improved, and the SACP0507-0.5 Ga had the minimal weight gain, illustrating that its improvement in the oxidation resistance properties was the most significant. It was worthwhile that the best ingredient point was in agreement with the wettability, further proving the relationship between the oxidation resistance and the wettability of the solder. The addition of Ga could not only increase the oxidation resistance ability but also reduce the surface tension of the solders. As a result, after adding proper amount of $\mathrm{Ga}$, the wettability improved. However, when there was too much Ga, the Ga-rich phase would lead to the uneven distribution of $\mathrm{Ga}$ in the surface, so that the $\mathrm{O}$ atoms could be in contact with the solder, worsening the oxidation resistance properties.

\section{Conclusions}

A small amount of element Ga could effectively improve the microstructure of the solder matrix in $\mathrm{Sn}-0.5 \mathrm{Ag}-0.7 \mathrm{Cu}-$ $0.05 \mathrm{Pr}$ lead-free solders. The structure gradually refined and the IMC in coarse particles became small and evenly distributed. When the addition amount of $\mathrm{Ga}$ was about $0.5 \%$, the effect was the most obvious.
The wettability of the solder was improved with the increasing content of $\mathrm{Ga}$ and reached the maximum when the amount was $0.5 \%$. Then, with the continuing increase in Ga content, the wetting properties showed a slight downward trend. This was mainly because Ga could be enriched in the solder surface so as to reduce the surface tension of the solder. However, with excess $\mathrm{Ga}$, the solder wettability was reduced owing to the formed $\mathrm{Ga}$-rich phase.

The influence of $\mathrm{Ga}$ in the oxidation resistance was similar to that in wettability, namely, the molten solder became relatively bright with proper $\mathrm{Ga}$ addition and the oxidation resistance increased and achieved the best at $0.5 \% \mathrm{Ga}$. But as the content of Ga continued to increase, there was oxide film on the solder surface. The oxidation rate with time slowed down due to the blocking role of the formed oxidation layer.

\section{Disclosure}

An earlier version of this work was presented as an abstract at Joining of Advanced and Specialty Materials (JASM XVII), Materials Science \& Technology, 2015. 


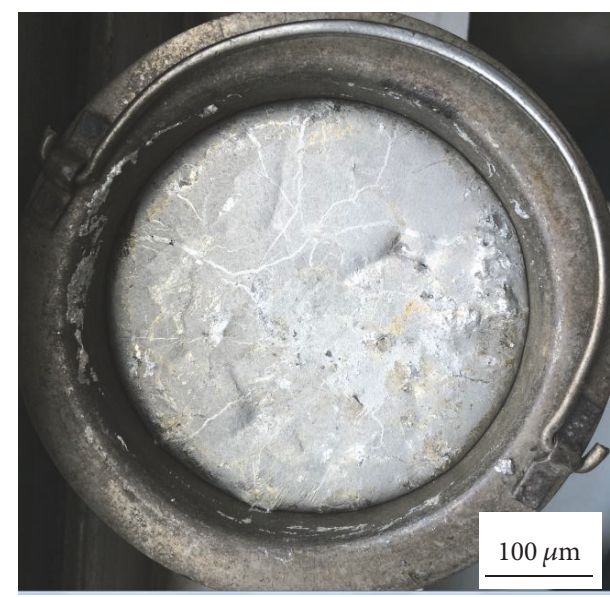

(a) SACP-0Ga

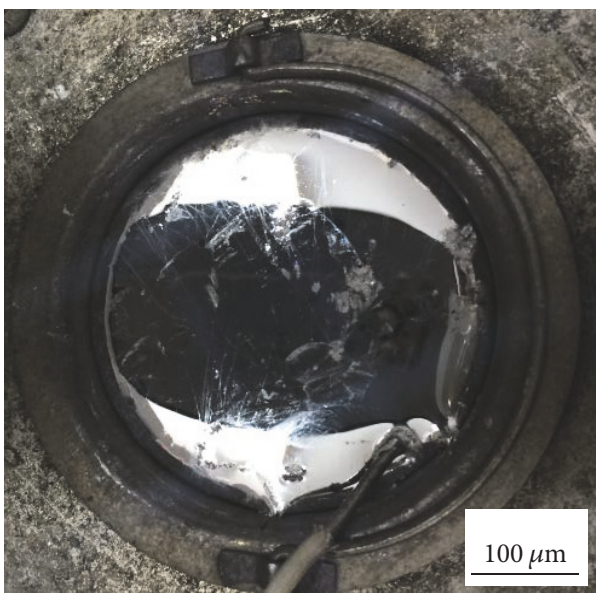

(c) SACP- $0.5 \mathrm{Ga}$

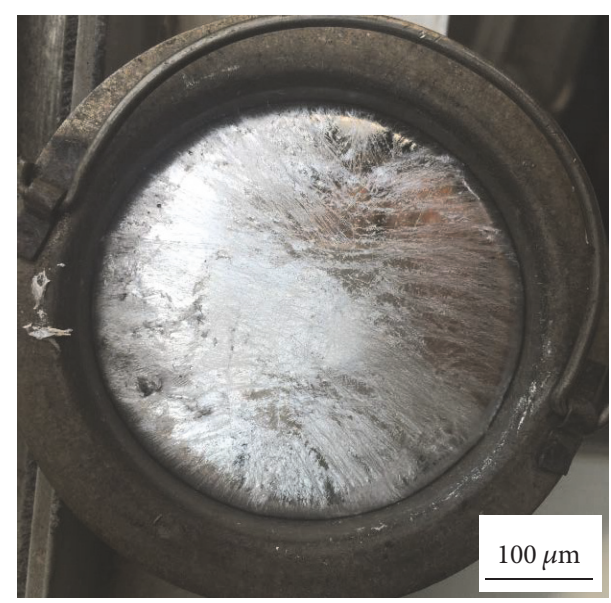

(b) SACP-0.1Ga

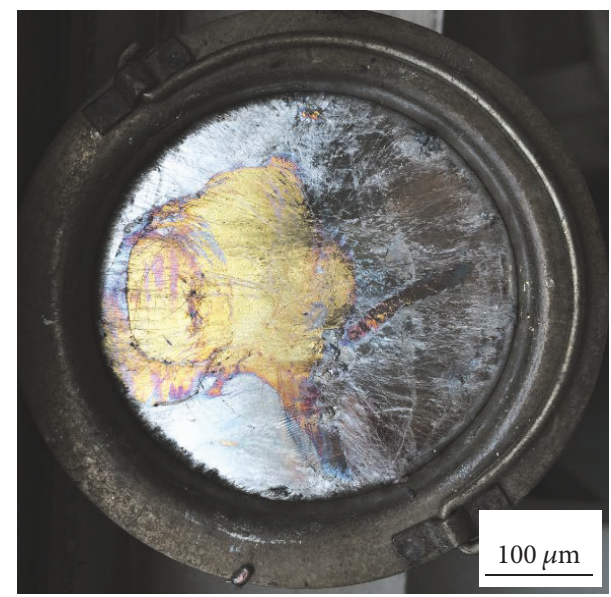

(d) SACP-1Ga

FIgURE 7: The surface morphology photographs of solders after incubation at $245^{\circ} \mathrm{C}$ for $30 \mathrm{~min}$.

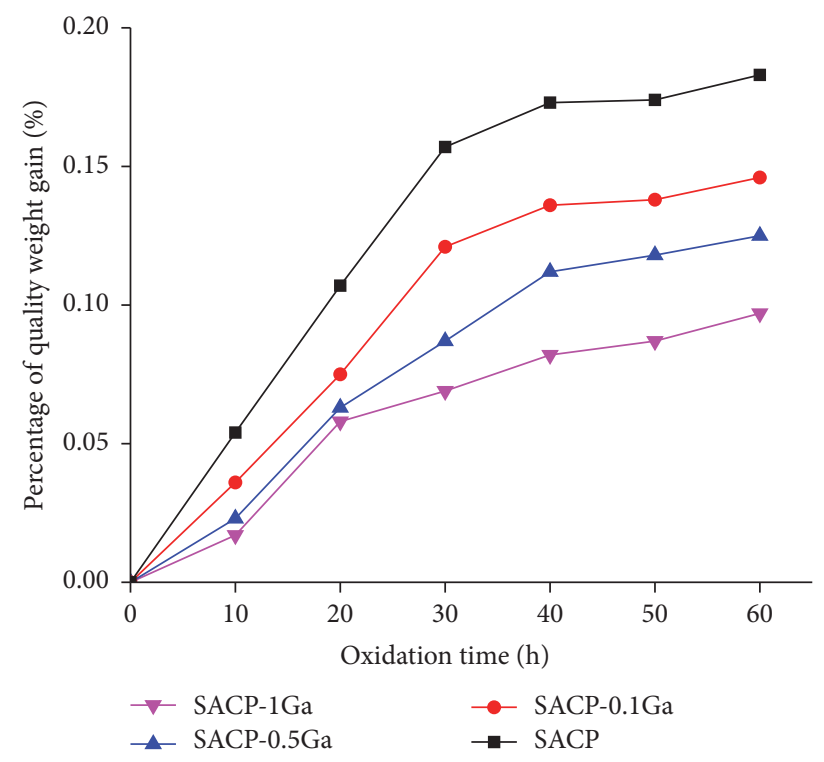

FIGURE 8: The oxidation weight gain curves of the solders.

\section{Conflicts of Interest}

The authors declare that there are no conflicts of interest regarding the publication of this paper.

\section{Acknowledgments}

This work was supported by the Key Laboratory of Advanced Welding Technology of Jiangsu Province, China (JSAWT-1404). This work was also supported by the Priority Academic Program Development of Jiangsu Higher Education Institutions.

\section{References}

[1] Z. Xiao, S. Xue, Y. Hu, H. Ye, L. Gao, and H. Wang, "Properties and microstructure of Sn-9Zn lead-free solder alloy bearing Pr," Journal of Materials Science: Materials in Electronics, vol. 22, no. 6, pp. 659-665, 2011. 
[2] J. Zhao, L. Qi, X.-M. Wang, and L. Wang, "Influence of Bi on microstructures evolution and mechanical properties in Sn-Ag$\mathrm{Cu}$ lead-free solder," Journal of Alloys and Compounds, vol. 375, no. 1-2, pp. 196-201, 2004.

[3] K. S. Kim, S. H. Huh, and K. Suganuma, "Effects of intermetallic compounds on properties of $\mathrm{Sn}-\mathrm{Ag}-\mathrm{Cu}$ lead-free soldered joints," Journal of Alloys and Compounds, vol. 352, no. 1-2, pp. 226-236, 2003.

[4] T. H. Chuang, M. W. Wu, S. Y. Chang, S. F. Ping, and L. C. Tsao, "Strengthening mechanism of nano- $\mathrm{Al} 2 \mathrm{O} 3$ particles reinforced Sn3.5Ag0.5Cu lead-free solder," Journal of Materials Science: Materials in Electronics, vol. 22, no. 8, pp. 1021-1027, 2011.

[5] D. A.-A. Shnawah, S. B. M. Said, M. F. M. Sabri, I. A. Badruddin, and F. X. Che, "Novel Fe-containing Sn-1Ag-0.5Cu lead-free solder alloy with further enhanced elastic compliance and plastic energy dissipation ability for mobile products," Microelectronics Reliability, vol. 52, no. 11, pp. 2701-2708, 2012.

[6] D. X. Luo, S. B. Xue, and Z. Q. Li, "Effects of Ga addition on microstructure and properties of $\mathrm{Sn}-0.5 \mathrm{Ag}-0.7 \mathrm{Cu}$ solder," Journal of Materials Science: Materials in Electronics, vol. 25, no. 8, pp. 3566-3571, 2014.

[7] D.-X. Luo, S.-B. Xue, and S. Liu, "Investigation on the intermetallic compound layer growth of $\mathrm{Sn}-0.5 \mathrm{Ag}-0.7 \mathrm{Cu}-\mathrm{xGa} / \mathrm{Cu}$ solder joints during isothermal aging," Journal of Materials Science: Materials in Electronics, vol. 25, no. 12, pp. 5195-5200, 2014.

[8] A. E. Hammad, "Investigation of microstructure and mechanical properties of novel $\mathrm{Sn}-0.5 \mathrm{Ag}-0.7 \mathrm{Cu}$ solders containing small amount of Ni," Materials \& Design, vol. 50, pp. 108-116, 2013.

[9] J. Glazer, "Microstructure and mechanical properties of $\mathrm{Pb}$-free solder alloys for low-cost electronic assembly: a review," Journal of Electronic Materials, vol. 23, no. 8, pp. 693-700, 1994.

[10] L. Zhang, S.-B. Xue, L.-L. Gao et al., "Microstructure characterization of $\mathrm{SnAgCu}$ solder bearing Ce for electronic packaging," Microelectronic Engineering, vol. 88, no. 9, pp. 2848-2851, 2011.

[11] A. A. El-Daly and A. M. El-Taher, "Evolution of thermal property and creep resistance of $\mathrm{Ni}$ and $\mathrm{Zn}$-doped $\mathrm{Sn}-2.0 \mathrm{Ag}-0.5 \mathrm{Cu}$ lead-free solders," Materials \& Design, vol. 51, pp. 789-796, 2013.

[12] P. Gao, J. Lin, Y. Lei, and G. Wen, "Drop performance research of silver content on the $\mathrm{SnAgCu}$ based lead-free solder of joint level," in Proceedings of the 2013 14th International Conference on Electronic Packaging Technology, ICEPT 2013, pp. 809-813, chn, August 2013.

[13] Y. Liu, F. Sun, and X. Li, "Effect of Ni, Bi concentration on the microstructure and shear behavior of low-Ag SAC-Bi-Ni/Cu solder joints," Journal of Materials Science: Materials in Electronics, vol. 25, no. 6, pp. 2627-2633, 2014.

[14] T. J. Anderson and I. Ansara, "The Ga-Sn (gallium-tin) system," Journal of Phase Equilibria, vol. 13, no. 2, pp. 181-189, 1992.

[15] W. Chen, S. Xue, H. Wang, J. Wang, and Z. Han, "Investigation on properties of Ga to Sn-9Zn lead-free solder," Journal of Materials Science: Materials in Electronics, vol. 21, no. 5, pp. 496502, 2010.

[16] X. Ren, M. Li, and D. Mao, "Effect of alloying elements on the high-temperature oxidation resistance of $\mathrm{Sn}$ - Zn based lead-free solder," Electronic Components and Materials, vol. 11, article 015, 2014.

[17] P. Xue, S.-B. Xue, Y.-F. Shen, F. Long, and H. Zhu, "Mechanism of reaction between $\mathrm{Nd}$ and $\mathrm{Ga}$ in $\mathrm{Sn}-\mathrm{Zn}-0.5 \mathrm{Ga}-\mathrm{xNd}$ solder," Journal of Electronic Materials, vol. 43, no. 9, pp. 3404-3410, 2014. 

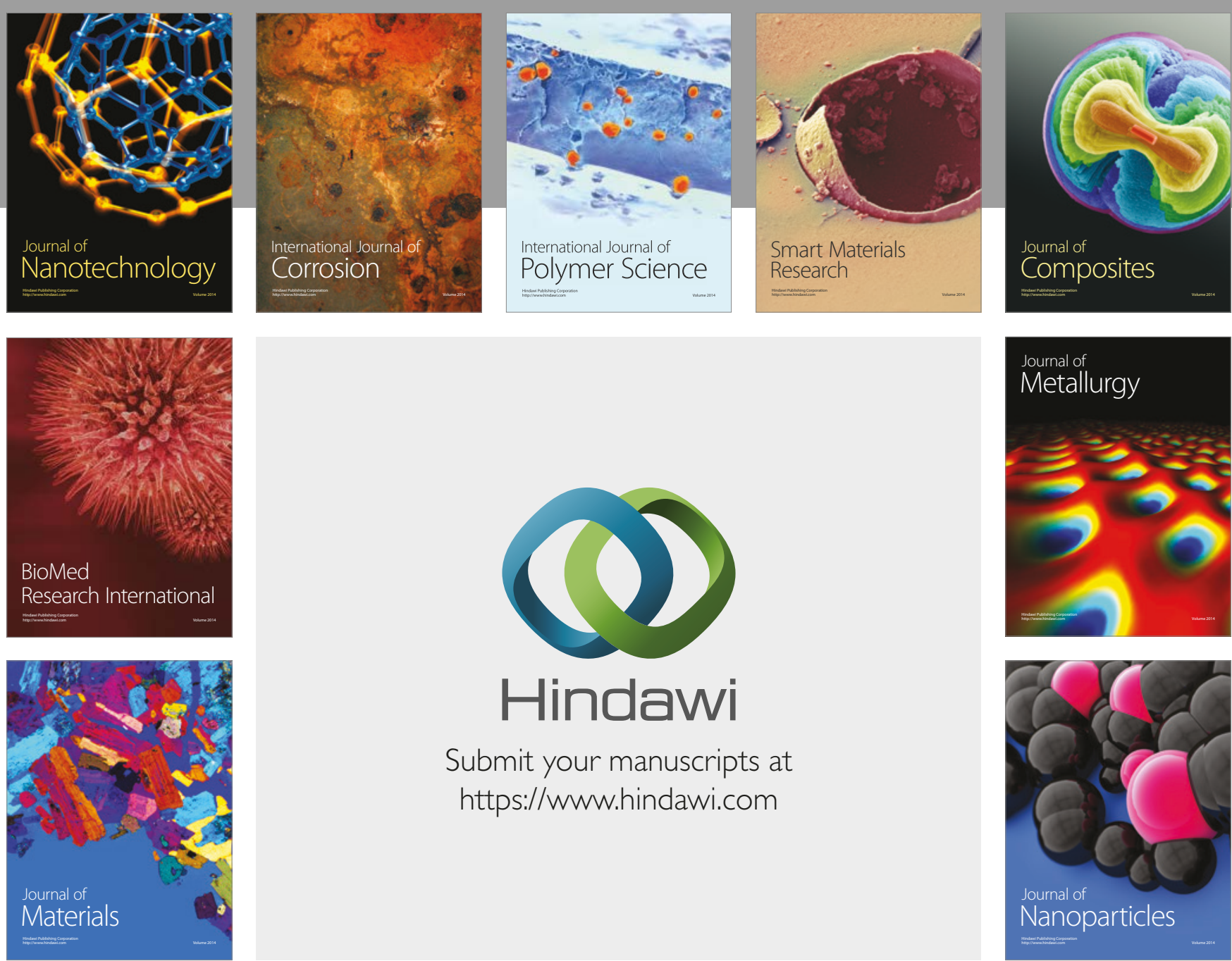

\section{Hindawi}

Submit your manuscripts at

https://www.hindawi.com
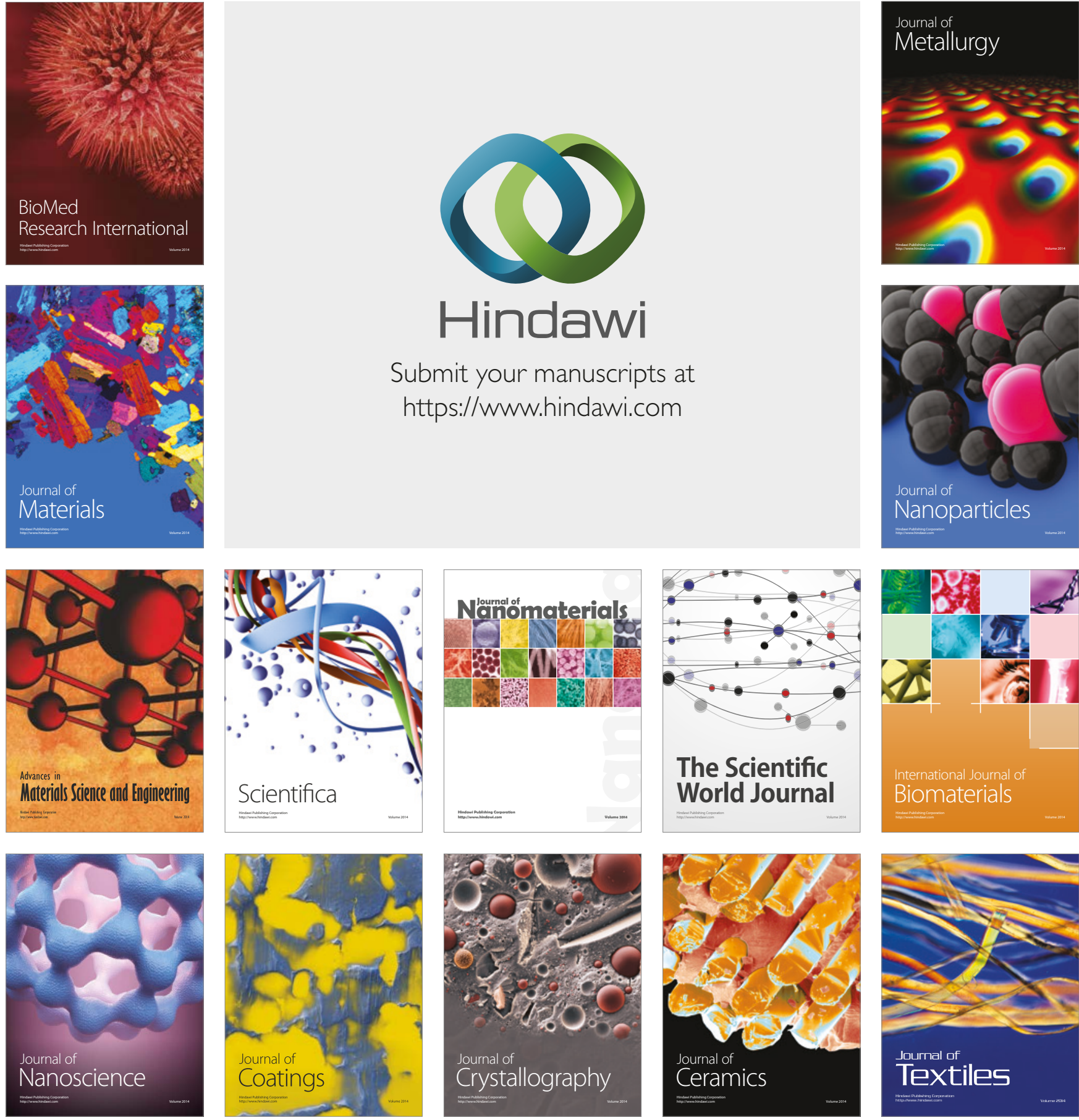

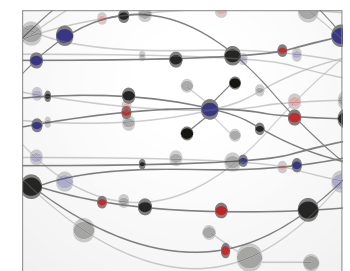

The Scientific World Journal
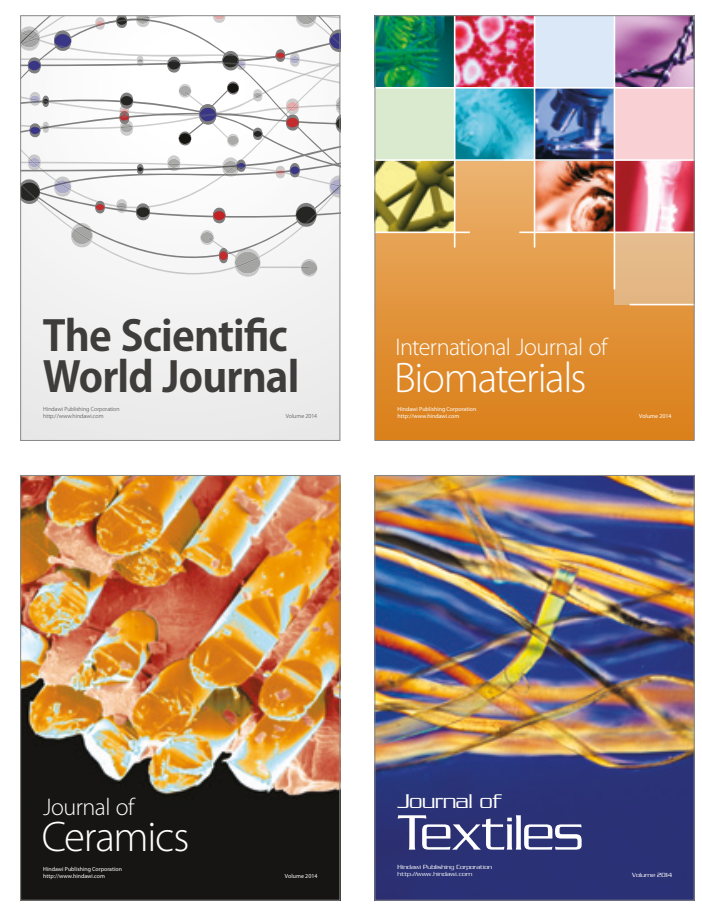